\title{
Bafilomycin A1 induces caspase-dependent apoptosis and in- hibits autophagy flux in diffuse large B cell lymphoma
}

\author{
Fang Li ${ }^{1,2}$, Ying $\mathrm{Hu}^{1}$, Yizhou Hu${ }^{3}$, Ren $\mathrm{Zhou}^{1}$, Zhengrong Mao ${ }^{1, *}$ \\ 1 Department of Pathology and Pathophysiology, Institute of Pathology and Forensic Medicine, Zhejiang \\ University School of Medicine, Hangzhou, China.; 21718498@zju.edu.cn (F,L); 18757262623@163.com (Y,H); \\ zhouren@zju.edu.cn $(\mathrm{R}, \mathrm{Z})$ \\ 2 Laboratory of Cancer Biology, Key Lab of Biotherapy in Zhejiang, Sir Run Run Shaw Hospital, Medical \\ school of Zhejiang University, Hangzhou, China \\ 3 Zhejiang University School of Medicine, Hangzhou, China; hyzhou3@gmail.com \\ * Correspondence: maozr@zju.edu.cn
}

\begin{abstract}
Diffuse large B cell lymphoma (DLBCL), the most frequent type of non-Hodgkin lymphoma in adulthood, remains challenging clinical issues. Despite enhanced remission rates can be achieved, there are one-third of patients who will not respond to current treatment or will relapse with resistant disease, necessitating ongoing efforts on effective treatment strategies and agents. The vacuole $\mathrm{H}^{+}$-ATPase inhibitor bafilomycin A1 is broadly used to block late stage of autophagy flux at high concentration. In this study, we show that, to our surprise, bafilomycin A1 effectively inhibited and killed DLBCL cells at nanomolar concentrations (5nM). Bafilomycin A1 targeted cell cycle regulators cyclin D1 and cyclin E2 to induce cell cycle arrest in G0/G1 phase. Meanwhile, it induced caspase-dependent apoptosis with concomitant cleaved caspase-3 and Parp. Furthermore, we found that bafilomycin A1 inhibited autophagy flux at both early and late stages of the autophagy flux through activating ERK and mammalian target of rapamycin signaling, as well as by inhibiting the degradation of autolysosomes. We speculated that bafilomycin A1 as autophagy inhibitor might enhance the effect of DLBCL chemotherapeutic drug rituximab. Accordingly, our results provided evidence that the combination of bafilomycin A1 with rituximab enhanced the inhibition of DLBCL cells notably. Taken together, our data suggest that bafilomycin A1 may be a promising candidate drug in the therapy of diffuse large B cell lymphoma.
\end{abstract}

Keywords: apoptosis; autophagy; bafilomycin A1; diffuse large B cell lymphoma

\section{Introduction}

Diffuse large B cell lymphoma (DLBCL) is the most prevalent type of aggressive nonHodgkin lymphoma (NHL) in adulthood worldwide [1], which can arise de novo or from clinical evolution of indolent B cell lymphomas, such as follicular lymphoma (FL) and chronic lymphocytic leukemia (CLL) [2]. DLBCL represents a heterogeneous set of diseases with differed outcomes that are characterized by clinical features, cell of origin (COO), molecular features, and frequently recurring mutations [3]. A giant advancement was progressed by application of gene expression profiling (GEP) which provided a basis for subdividing cases into group [4]. The prevalent dividing system classifies DLBCL cases judging by cell-of-origin into germinal center B-cell like (GCB) and activated B-cell like (ABC) subtypes [5], with 10-15\% of cases remaining unclassifiable. Patients with GCB subtype have a better prognosis than patients with non-GCB subtype (ABC and unclassifiable) [6]. The current 5-year overall survival rate is $60 \%-70 \%$ using frontline therapy RCHOP (rituximab, cyclophosphamide, doxorubicin, vincristine, and prednisone) [7-8]. Although durable remissions can be achieved in the major proportion of cases $(>50 \%)$ by up-front chemoimmunotherapy $(\mathrm{CI})$, still there are one-third of patients who will not 
respond to standard regimens or will relapse with resistant disease [9-11]. Thus, effective and novel treatment strategies and agents are urgently needed to improve the therapeutic outcome of DLBCL.

Bafilomycin A1, a macrolide antibiotic which derived from Streptomyces griseus, is an inhibitor of vacuolar $\mathrm{H}^{+}$ATPase (V-ATPase) [12-13]. Bafilomycin A1 targets the V0 sector subunit of V-ATPase complex and inhibit rotation and passage of protons into the lysosomal lumen, inhibiting $\mathrm{H}^{+}$translocation, therefore reducing acidification of vesicles such as endosomes, lysosomes and phagosomes [14]. Bafilomycin A1 has been used as inhibitor of late stage of autophagy frequently, blocking fusion between autophagosomes and lysosomes [15]. Besides the above effect, bafilomycin A1 was found to inhibit the growth of variable cancer cells under hypoxic conditions by expressing hypoxia-inducible factor1alpha (HIF-1 $\alpha$ ) [16-17]. Moreover, recent study has demonstrated that bafilomycin A1 targets both autophagy and apoptosis pathways in pediatric B-cell acute lymphoblastic leukemia [18]. In addition, one investigation suggests that bafilomycin A1 induces caspase-independent cell death in hepatocellular carcinoma cells via targeting of autophagy and MAPK pathways [19]. However, usually bafilomycin A1 is used at high concentrations $(0.1-1 \mu \mathrm{M})$ and are associated with adverse effects due to acidosis and hypoxia which also occur in normal cells in physiological conditions [20-22], therefore bafilomycin A1 is hindered its application and clinical trials.

Autophagy, (hereafter referred to as autophagy), is a conservative cellular catabolic pathway which maintains homeostasis and sustain metabolic stress by the turnover of proteins and organelles and degradation of lysosomes [23-25]. Cytoplasmic contents such as damaged, misfolded proteins and damaged organelles are sequestered into doublemembrane vesicles which is called autophagosomes and degraded by the fusion of autophagosomes with lysosomes when single-membrane autolysosomes is created [26-30]. In a wide range of cancer cells, autophagy is observed while its function differs under variable cancer subtype and cellular context [31-33]. Similarly, in hematological malignancies, autophagy can be a double-edged sword, either acting as a chemo-resistant role or tumor suppressive role, which depends on the cellular context [18]. Among blood cancers, autophagy may act as cytoprotective roles and remove stimuli from standard treatments then result in chemotherapy resistance. In colorectal cancer, one study suggests that the combination of Apatinib with autophagy inhibitor chloroquine (CQ) tends to have the most significant anti-tumor effect both in vitro and in vivo [34]. As well as in pancreatic cancer, one investigation shows that autophagy inhibitor chloroquine synergistically enhanced the ability of ERK inhibitors to mediate the antitumor activity in KRAS-driven PDAC [35]. In sum, the combination of autophagy inhibitors with specific signaling pathway inhibitors or standard chemotherapy drugs may an effective strategy for cancer.

In this study, we hypothesized that autophagy inhibitor bafilomycin A1 may exert a tumor suppression function in DLBCL. Here, we present data demonstrating that bafilomycin A1 effectively inhibits and kills DLBCL cell at a low concentration. Further experiments revealed that bafilomycin A1 functions as a tumor suppressor by attenuating cytoprotective autophagy via multiple targets and inducing caspase-dependent apoptosis. We then exploited that DLBCL chemotherapy rituximab synergistically enhanced bafilomycin A1-mediated inhibition of DLBCL cells growth. Our finding have validated that bafilomycin A1 may be a potential therapeutic drug for the treatment of DLBCL.

\section{Materials and Methods}

\subsection{Cell culture}

DLBCL cell lines OCI-ly10, OCI-ly19, SUDHL-2, SUDHL-4, Pfeiffer and Toledo were obtained from the Type Culture Collection of the Chinese Academy of Sciences (Shanghai, China). The DLBCL cell lines were maintained in IMDM with 10\% fetal bovine serum (FBS)(Gibco). Cells were cultured in a $5 \% \mathrm{CO}_{2}$ humidified incubator at $37^{\circ} \mathrm{C}$. 


\subsection{Cell proliferation}

The cytotoxicity of bafilomycin A1 on DLBCL cells was estimated by Cell Counting Kit-8 (CCK-8 assay). As previously described, cells $\left(1 \times 10^{4} /\right.$ well $)$ were plated into 96 -well plates in triplicates while cultured in $100 \mu \mathrm{L}$ IMDM containing 10\% FBS before the start of treatment. Then cells were treated with bafilomycin A1 at 0.5, 1, 5, 10, 20nM for 24h. Then cells were treated with bafilomycin A1 at $5 \mathrm{nM}$ for 24, 48, 72, $96 \mathrm{~h}$. $10 \mu \mathrm{L}$ CCK-8 solutions was added to each plate and cells were incubated for $3 \mathrm{~h}$ at $37^{\circ} \mathrm{C}$. Cell viability was measured at 450nM on SynergyMx M5.

\subsection{Cell cycle}

Cells were treated with control or bafilomycin A1 for $24 \mathrm{~h}$ and then collected and fixed in cold absolute ethanol overnight at $4^{\circ} \mathrm{C}$. Cells were treated with PBS (contain $100 \mu \mathrm{g} / \mathrm{mL} \mathrm{RNase} \mathrm{A,} 50 \mu \mathrm{g} / \mathrm{mL}$ PI) and then incubated for $15 \mathrm{~min}$ at room temperature in the darkness. Samples were analyzed by flow cytometry.

\subsection{Immunoblotting}

Cells were harvested and subjected to lysis in 1xSDS loading buffer and then resolved by SDS-polyacrylamide gel electrophoresis (PAGE), transferred to PVDF membranes and blocked in Bovine Serum Albumin (BSA) in Tris Buffered Saline (TBS) with 0.1\% Tween20. PVDF membranes were incubated with primary antibodies at $4^{\circ} \mathrm{C}$ overnight. The secondary antibodies were used at a concentration of 1:10000. The proteins were visualized by Odyssey CLx. (LI-COR America).

\subsection{Apoptosis assay}

Cells were treated with control or bafilomycin A1 for $24 \mathrm{~h}$ and then collected and stained with fluorescein isothiocyanate (FITC)-conjugated annexin $\mathrm{V}$ and propidium iodide (PI), then incubated for $15 \mathrm{~min}$ at room temperature. Samples were analyzed by flow cytometry to detect apoptosis.

\subsection{Autophagic flux assay and confocal microscopy}

Adenovirus-mCherry-GFP-LC3B was obtained from Beyotime (C3011) and the admCherry-GFP-LC3B was infected into DLBCL cell lines. DLBCL cell lines were subjected to EBSS, bafilomycin A1. After $24 \mathrm{~h}$, the fluorescence images were captured by confocal high-resolution microscope (OLYMPUS IX83-FV3000-OSR) and the autophagosomes (yellow dots) and autolysosomes (only red dots) were counted.

\subsection{Transmission electron microscopy}

DLBCL cells were fixed in $2.5 \%$ glutaraldehyde at $4^{\circ} \mathrm{C}$ overnight then embedded in embedding medium. Thin sections were examined by transmission electron microscope (Tecnai G2 F20 S-TWIN FEI). Autophagosomes were defined as double-membrane vacuoles measuring 0.1 or $1.0 \mu \mathrm{m}$.

\subsection{Statistical analysis}

Data are expressed as the mean \pm standard deviation of at least three independent experiments. Two-sided t-test was used to compare the differences between groups. $\mathrm{P}$ value $<0.05$ were considered statistically significant. Graph Pad Prism 6.01 software was carried out for all statistical analysis. $\left({ }^{*} \mathrm{P}<0.05,{ }^{* *} \mathrm{P}<0.01,{ }^{* * *} \mathrm{P}<0.001,{ }^{* * * *} \mathrm{P}<0.0001\right)$ 


\section{Results}

\subsection{Bafilomycin A1 inhibits the growth of DLBCL cells.}

Bafilomycin A1, a specific inhibitor of vacuolar-type H+ ATPase (V-ATPase), is often used to inhibit autophagy flux at concentration between 0.1 and $1 \mathrm{uM}$. While studying how autophagy works in DLBCL, we observed an unexpected sensitivity of DLBCL cells to bafilomycin A1, which lead us to investigate the effect of bafilomycin A1 on DLBCL cell lines. DLBCL cell lines including OCI-ly10, OCI-ly19 were cultured in the presence of increasing concentrations of bafilomycin A1(0.5 nM, 1 nM, 5 nM, 10 nM, 20 nM). Cell viability was measured by CCK8. The results showed that different concentrations of bafilomycin A1 inhibits the growth of OCI-ly10 and OCI-ly19 cell in different degrees. The minimum concentration of bafilomycin A1 which works on both cell types is $5 \mathrm{nM}$. As such, bafilomycin A1 at $5 \mathrm{nM}$ was used in subsequent experiments. (Figure 1A) DLBCL cell lines OCI-ly10, OCI-ly19, SUDHL-2, SUDHL-4, Toledo and Pfeiffer were treated with $5 \mathrm{nM}$ bafilomycin A1 for 24-96h. CCK8 assays were carried out to determine the growth kinetics of the cell lines. As shown in Figure 1B, all cell lines were significantly inhibited with the treatment of $5 \mathrm{nM}$ bafilomycin A1 for 96 h, OCI-ly10, OCI-ly19, SUDHL-2, SUDHL-4 were inhibited by $5 \mathrm{nM}$ bafilomycin A1 significantly since $24 \mathrm{~h}$ (Figure 1B). To dissect the mechanism of bafilomycin A1 suppressing DLBCL cell growth, we performed flow cytometric analysis of propidium iodide (PI)-stained samples of DLBCL cell lines cultured for $24 \mathrm{~h}$ in the existence of $5 \mathrm{nM}$ bafilomycin A1. Bafilomycin A1 increased the percentage of SUDHL-2 cells in the G0/G1 phase of the cell cycle, with a synchronous decrease in $S$ and G2/M phases of cells, revealing a G0/G1 phase arrest (Figure 1C). To further expound how bafilomycin A1 reins in cell cycle progression, we examined the protein levels of key regulators of cell cycle in OCI-ly10, SUDHL-2, SUDHL-4, Pfeiffer cells. Exposed to bafilomycin A1 induced a reduction in G1 phase regulator Cyclin D1, while Cyclin E2 increased after the treatment of bafilomycin A1 (Figure 1D). The above data suggest that bafilomycin A1 may induce G0/G1 cell cycle arrest by down-regulating positive regulators of the cell cycle. 


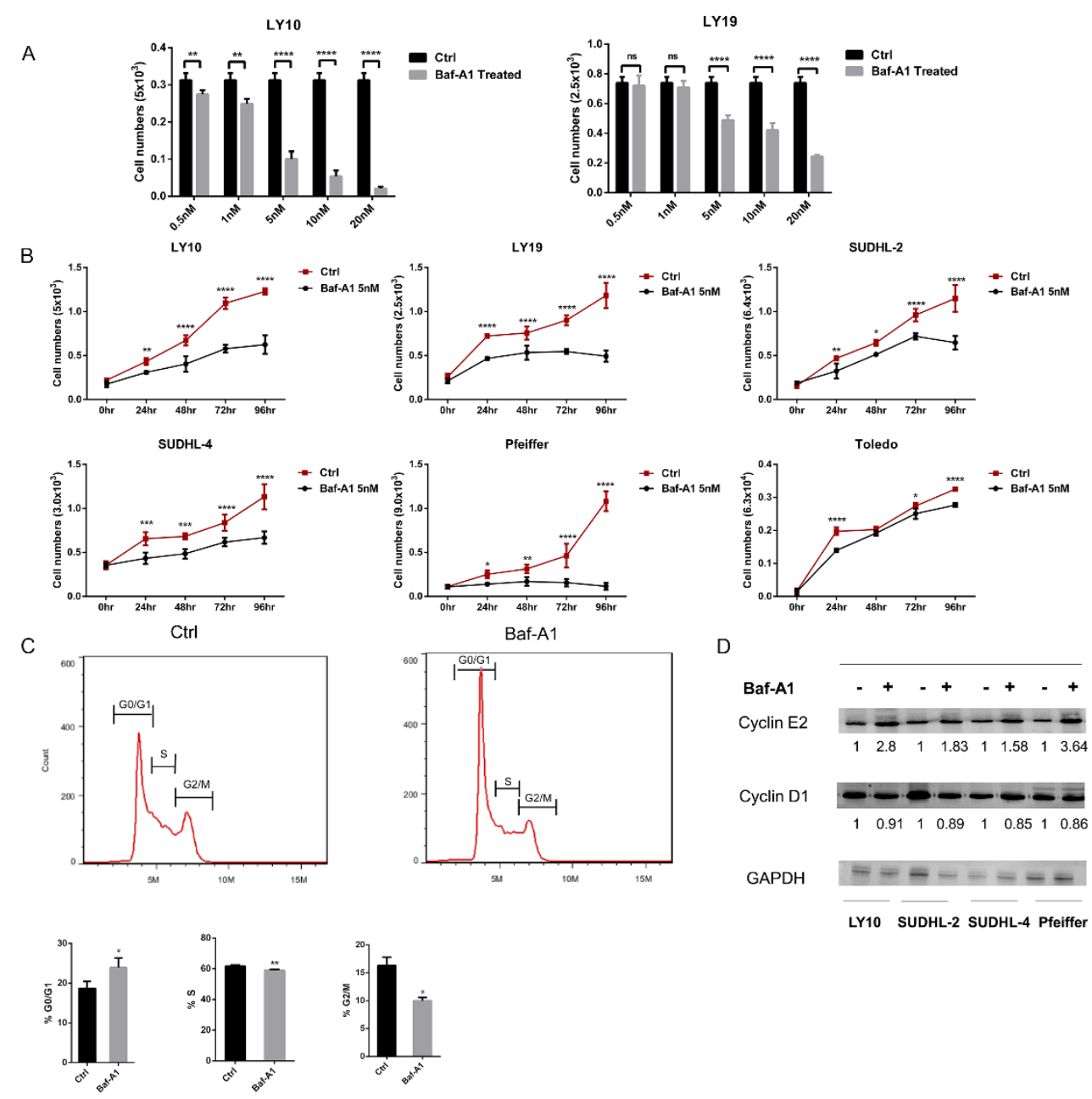

Figure 1. Bafilomycin A1 inhibits the growth of DLBCL cells. (A) Effect of bafilomycin A1 on the growth of DLBCL cell lines measured by CCK8 assay. DLBCL cell lines were cultured for $24 \mathrm{~h}$ with increasing levels of bafilomycin A1. $5 \mathrm{nM}$ Bafilomycin A1 can inhibit the growth of DLBCL cell lines significantly at $24 \mathrm{~h}$. (B) Effect of bafilomycin A1 on the growth of DLBCL cell lines measured by CCK8 assay. Bafilomycin A1 ( $5 \mathrm{nM})$ inhibits the growth of DLBCL cells over $96 \mathrm{~h}$. (C) Effect of bafilomycin A1 on the cell cycle of DLBCL cells. The DLBCL cells were treated with bafilomycin A1, the cell cycle was analyzed by flow cytometry after PI staining. Bafilomycin A1 increased the percentage of cells in the G0/G1 phase and decreased the percentage of cells in the S and G2/M phases of the cell cycle. The representative histogram data and the percentage of cells in each stage of the cell cycle after $24 \mathrm{~h}$ of treatment are shown. (D) Effect of bafilomycin A1 on cell cycle regulators evaluated by western blotting analysis and qPCR. The numbers below the blots indicate relative intensity of Cyclin E2, Cyclin D1 relative to GAPDH. The decreased expression of Cyclin D1 inhibits the transition from G0/G1 to $S$ in cell cycle. ${ }^{* *} \mathrm{P}<0.001$, ${ }^{* *} \mathrm{P}<0.01$.

\subsection{Bafilomycin A1 triggers caspase-dependent apoptosis of DLBCL cells.}

To determine whether bafilomycin A1 inducing DLBCL cells inhibition resulted from cell death, bafilomycin A1-treated SUDHL-2 and SUDHL-4 cells were analyzed by flow cytometry with PI-conjugated Annexin-V double staining. As illustrated in Figure 2A, bafilomycin A1 increased the percentage of both early and late apoptotic cells in both SUDHL-2 and SUDHL-4 cell lines, suggesting bafilomycin A1-mediated induction of 
apoptotic cell death (Figure 2A). Meanwhile, two classical apoptosis markers, caspase-3 activation and Poly (ADP-ribose) polymerase (PARP) cleavage, were observed in bafilomycin A1-treated SUDHL-2 cells for 72 h. Cleaved caspase-3 occurred, with concomitant occurrence of cleaved PARP for $72 \mathrm{~h}$ (Figure 2B).

A

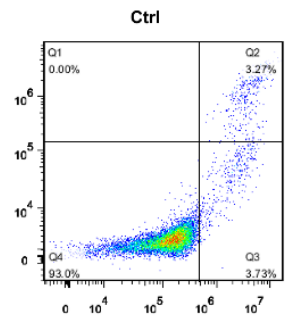

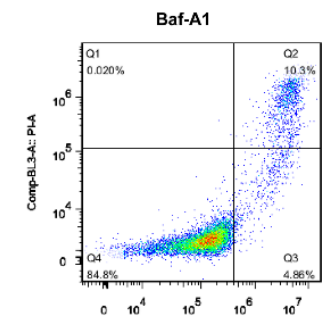

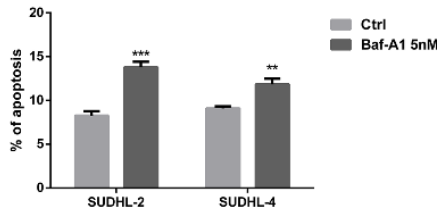

B

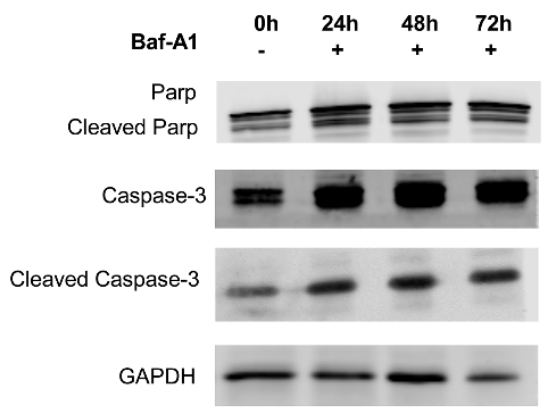

Figure 2. Bafilomycin A1 triggers caspase-dependent apoptosis of DLBCL cells. (A) Bafilomycin A1 induces apoptosis of DLBCL cell lines. DLBCL cells were cultured with or without bafilomycin A1 5nM for $24 \mathrm{~h}$ then were analyzed by flow cytometry after annexin VFITC/PI staining. Left, a representative flow cytometric plot for annexin V-FITC/PIstained DLBCL cells. Right, quantification of apoptotic cells of SUDHL-2, SUDHL-4 cells. (B) Western blot analysis of caspase-3 and Parp cleavage in DLBCL cell lines. The cells were treated with $5 \mathrm{nM}$ bafilomycin A1 for a specific period of time. ${ }^{* * *} \mathrm{P}<0.001$, ${ }^{* *} \mathrm{P}<0.01$.

\subsection{Bafilomycin A1 inhibits autophagy flux effectively of DLBCL cells at low concentration.}

Bafilomycin A1 is usually used as autophagy inhibitor at concentration between 0.1 and $1 \mathrm{uM}$, blocking autophagosome-lysosome fusion during the late stage of autophagy. We detected whether bafilomycin A1 at low concentration may still target at autophagy. OCI-ly10, SUDHL-2, SUDHL-4 and Pfeiffer were cultured in the presence of $5 \mathrm{nM}$ bafilomycin A1 for $24 \mathrm{~h}$, western blotting was performed to examine changes of autophagy marker proteins such as LC3 and P62. Degradation of p62 is generally used as an autophagy flux marker. As shown in Figure 3A, western blotting revealed that conversion of LC3-II from LC3-I increased upon bafilomycin A1 treatment, while P62 accumulated after $24 \mathrm{~h}$ (Figure 3A). Exposure of SUDHL-2 cells to bafilomycin A1 over $72 \mathrm{~h}$ induced robust LC3 conversion (i.e. cytosolic LC3-I to autophagosome-bound lipidated LC3-II (Figure 3B), suggesting bafilomycin A1 blocks basal autophagic flux in DLBCL cell lines. To confirm that bafilomycin A1 blocks basal autophagic flux, Ad-mCherry-GFP-LC3 was performed on SUDHL-2 cells. Ad-mCherry-GFP-LC3 is based on the finding that GFP, but not $\mathrm{mCherry}$ is quenched upon autophagic delivery of this protein to the acidic environment of lysosome. In this case, autophagosomes display both green and red fluorescence, while autolysosomes appear only red. Numbers of red-only (acidic) and red plus green (yellow, non-acidic) autophagic vesicles were assessed in SUDHL-2 cells infected with admCherry-GFP-LC3 subjected to bafilomycin A1 treatment. Bafilomycin A1 (5 nM) treatments induced a significant increase in the number of yellow puncta in SUDHL-2 compared with control group (Figure 3C). As anticipated, cultured with EBSS (Earle's 
Balanced Salts), a known solution as starvation treatment to induce autophagy, significantly increased the number of red autolysosomes in SUDHL-2 cells (Figure 3C). Together, these results indicate that bafilomycin A1, at low concentrations, block autophagy flux in late stage. Here are two possibilities, one is that bafilomycin A1 blocks autophagosome-lysosome fusion in SUDHL-2 cells; another is that bafilomycin A1 inhibits lysosome acidification, autophagosome and lysosome can still fuse but acidic environment inside lysosome impaired, the content of autolysosome will not be degraded. To dig deep in the mechanism of how bafilomycin A1 blocks autophagy flux in DLBCL cell lines, transmission electron microscopy (TEM) was performed to analyze the formation of double-membrane vesicles (autophagosomes) in bafilomycin A1-treated SUDHL-2 and SUDHL-4 cells. As shown in Figure 3D, autophagosomes in SUDHL-4 cells are most degradative autophagic vacuoles with double-membrane structures, the electronic cloud density inside vacuoles contents differs significantly from surroundings. The contents inside vacuoles increased after the treatment of bafilomycin A1 compared to control group (Figure 3D). As revealed in Figure 3E, autophagosomes in SUDHL-2 cells (control group) were mainly initial autophagic vacuoles holding transparent cleft, while vacuoles containing increased content such as lysosomes, multi-crests mitochondrial showed up in bafilomycin A1treated SUDHL-2 cells (Figure 3E). Taken together, bafilomycin A1 did not inhibit autophagosome-lysosome fusion but inhibit the acidification of lysosome to maintain $\mathrm{pH}$ inside lysosome high, misfolded proteins and impaired cellular organelles were not degraded. So was GFP fluorescence.

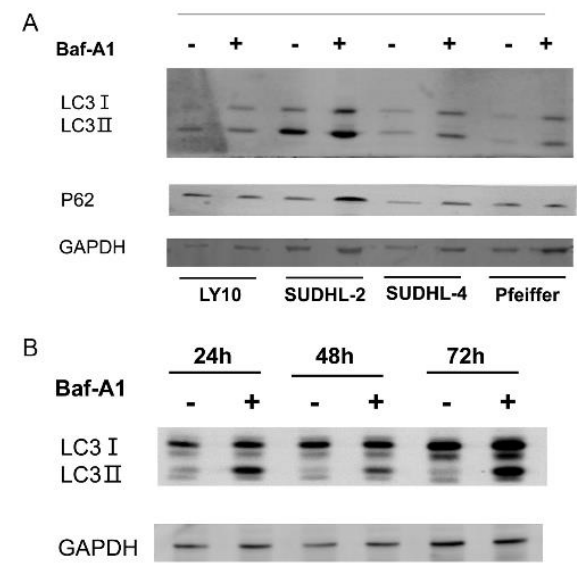

D

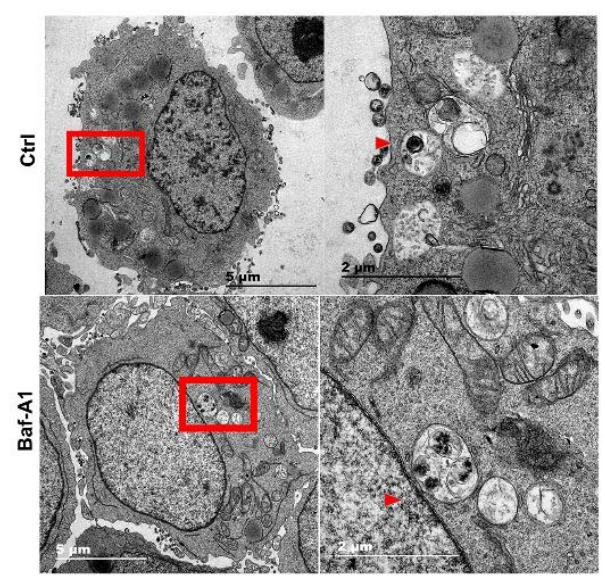

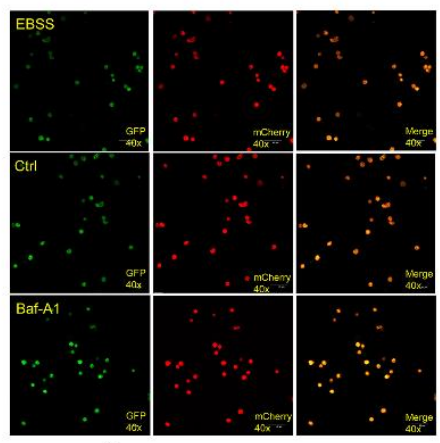

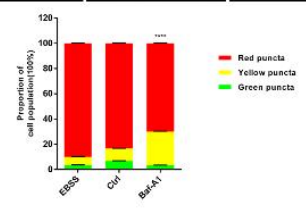

E

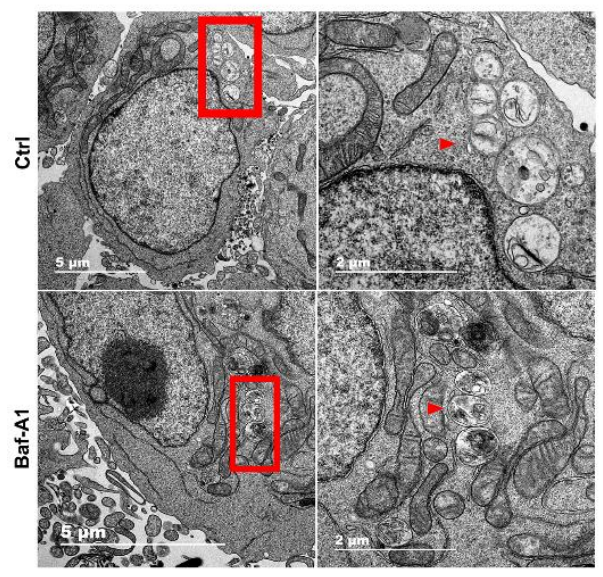


Figure 3. Bafilomycin A1 inhibits autophagy flux effectively of DLBCL cells at low concentration. (A) Western blot analysis of LC3, LC3 II , P62 levels in DLBCL cell lines. These cells were treated with $5 \mathrm{nM}$ bafilomycin A1 for $24 \mathrm{~h}$. (B) Western blot analysis of LC3, LC3 II levels in DLBCL cells. The cells were treated with $5 \mathrm{nM}$ bafilomycin A1 for a specific period of time. (C) Confocal microscopic analysis of autophagy flux with ad-mCherryGFP-LC3 fusion protein. Cells were infected with adenovirus for $48 \mathrm{~h}$ then treated with or without 5nM bafilomycin A1 for $24 \mathrm{~h}$. The yellow puncta indicates autophagosome, the red/orange puncta indicates autolysosome. The increasing yellow puncta after treatment with bafilomycin A1 indicates that autophagy flux is blocked. The numbers of cells with red puncta, yellow puncta and green puncta are shown. ${ }^{* * *} \mathrm{p}<0.0001$ versus ctrl (red puncta). (D) TEM images of autophagic vacuoles in SUDHL-4 cells. Cells were treated with bafilomycin A1 for $24 \mathrm{~h}$ then applied to TEM. Arrows indicate formed autophagosome or autolysosomes. The increasing autophagosome indicates that autophagy flux is blocked. (E) TEM images of autophagic vacuoles in SUDHL-2. Cells were treated with bafilomycin A1 for $24 \mathrm{~h}$ then applied to TEM. Arrows indicate formed autophagosome or autolysosomes. The increasing autophagosome indicates that autophagy flux is blocked.

\subsection{Bafilomycin A1 at low concentration effectively inhibits autophagy of DLBCL cell lines via multiple targets.}

We next examine the role of bafilomycin A1 in inhibiting autophagy flux in DLBCL cell lines. We already confirmed that bafilomycin A1 inhibits autolysosome degradation, given that mTOR/Erk signaling pathway involves in autophagy initiation, we detected key proteins involved in these pathways in bafilomycin A1-treated SUDHL-2 cells. Unexpectedly, we observed that bafilomycin A1 treatment up-regulated the phosphorylation levels of mTOR and Erk in a time-dependent manner (Figure 4A). No change was detected in the levels of total mTOR and Erk, suggesting bafilomycin A1 inhibits the early stage of autophagy by promoting Erk activation thus activating mTOR. Taken together, bafilomycin A1 not only inhibits autophagosome-lysosome fusion, but also inhibits the early stage of autophagy. The combined effects result in autophagy flux inhibition in DLBCL cell lines.

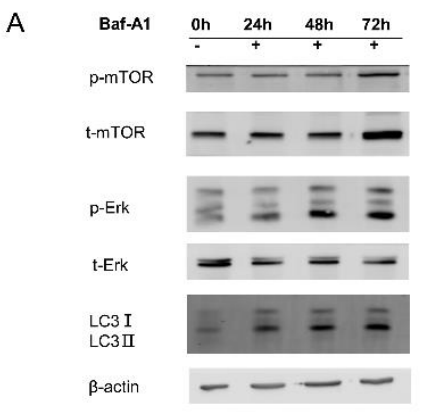

Figure 4. Bafilomycin A1 at low concentration effectively inhibits autophagy of DLBCL cell lines via multiple targets. Western blot analysis of p-mTOR, t-mTOR, p-Erk, t-Erk, LC3, LC3 II levels DLBCL cell lines. The cells were treated with or without 5nM bafilomycin A1 for $72 \mathrm{~h}$.

\subsection{Bafilomycin A1 enhances the tumor suppression effect of rituximab on DLBCL cell lines.}

Rituximab is a chimeric monoclonal antibody directed at CD20 with significant activity in non-Hodgkin lymphoma (NHL), nowadays the standard treatment of DLBCL is R$\mathrm{CHOP}$, in which $\mathrm{R}$ represents rituximab. We already showed the effect of bafilomycin A1 on DLBCL cell lines, that bafilomycin A1 triggers apoptosis. We hypothesized that 
bafilomycin A1 may enhance the tumor suppression effect of current chemotherapy-rituximab on DLBCL cells. To test this, OCI-ly10, OCI-ly19, SUDHL-2, SUDHL-4, Pfeiffer, Toledo cells were treated with control (IMDM), bafilomycin A1, rituximab or the combination of both bafilomycin A1 plus rituximab, CCK8 assay was performed to detect the proliferation of DLBCL cell lines. Consistent with our hypothesis, as shown in Figure 5A, treatment with bafilomycin A1 plus rituximab resulted in enhanced inhibition of OCI-ly10 cells, suggesting that bafilomycin A1 synergizes with rituximab to provide anti-proliferative effects on DLBCL cell lines (Figure 5A).

A

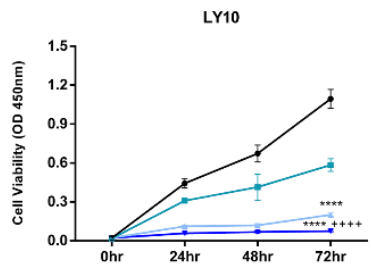

SUDHL-2
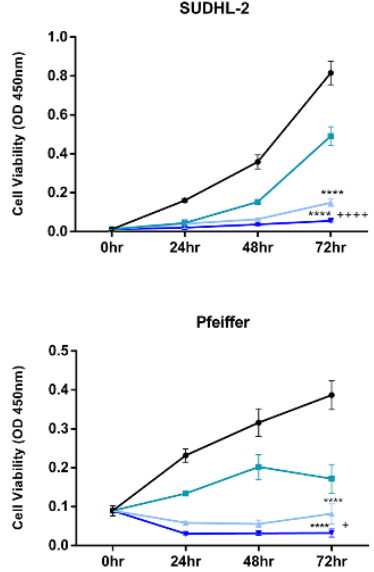
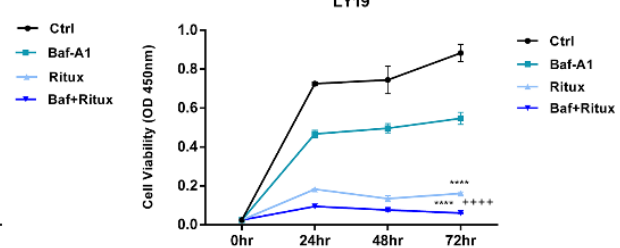

SUDHL-4
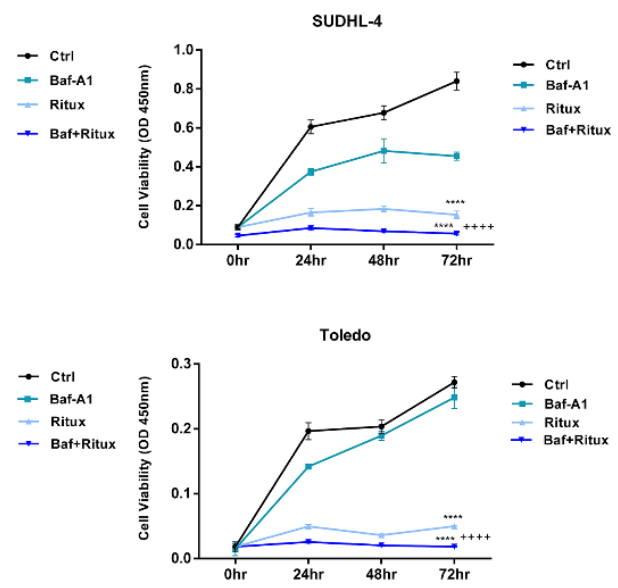

Figure 5. The growth of DLBCL cells was assessed over $72 \mathrm{~h}$ which were treated with: (1) IMDM (Control); (2) bafilomycin A1; (3) rituximab; (4) the combination of both agents. Center values are the mean; statistical testing was performed by two-sided t-test,

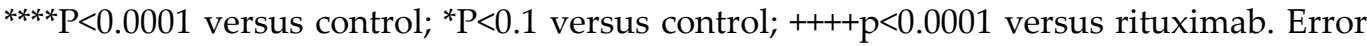
bars represent SD.

\section{Discussion}

Bafilomycin A1, as an inhibitor of V-ATPase, inhibits $\mathrm{H}^{+}$from translocating into vesicle lumen therefore suppresses the acidosis of organelles such as lysosomes and endosomes [36-37]. High concentrations of bafilomycin A1 (0.1-1 $\mathrm{MM})$ has been widely used to inhibit autophagy flux by blocking the fusion between autophagosomes and lysosomes which is a critical step in late-stage autophagy [38-39]. Meanwhile, bafilomycin A1 at high concentration exerts cytotoxicity in variable cancer such as hepatocellular carcinoma [19]. However, the high concentration of bafilomycin A1 may also induce severe cytotoxicity in normal cells, this hinders its use in broad ranges. Regarding the application of bafilomycin A1 in cancer, Yuan Na and colleagues have shown that bafilomycin A1 (1nM) effectively and specifically inhibited and killed pediatric B-cell acute lymphoblastic leukemia cells [18]. In agreement with previous studies, we have observed that bafilomycin A1 significantly inhibits the growth of DLBCL cell lines in low concentrations (0.5-20 nM). Besides this, the inhibitory effect of bafilomycin A1 on DLBCL cells was enhanced by the combination with DLBCL chemotherapy drug rituximab. Given that $30 \%$ of DLBCL patients are about to refractory to current chemotherapy or will relapse with resistant 
diseases, our finding suggest that bafilomycin A1 alone, or in combination with rituximab, may exert a promising therapeutic approach in DLBCL.

Several researchers have shown that bafilomycin A1 exerts an influence on cell cycle, causing cell cycle arrest in cancer cells [18-19]. Here, we have observed that after the treatment of bafilomycin A1 in DLBCL cells, bafilomycin A1 increased the percentage of DLBCL cells in the G0/G1 phase of the cell cycle, with a synchronous decrease in $S$ and G2/M phases of cells. In line with other studies, our date suggests a G0/G1 phase arrest in DLBCL cells cultured with bafilomycin A1. It's known that G0/G1 phase is mainly regulated by cyclin D1, high levels of cyclin D1 in G1 phase promote entry into S phase while levels of cyclin E2 drop greatly after entering S phase. In agreement with previous studies, we observed that cyclin D1 decreased, cyclin E2 accumulated in bafilomycin A1-induced cell cycle arrest, supporting that cyclin D1 and cyclin E2 play critical roles in inhibition of cell growth of bafilomycin A1. Thus, bafilomycin A1 targeted cell cycle regulator cyclin D1 and cyclin E2 to induce cell cycle arrest.

Targeting apoptosis has been the hit of killing DLBCL cells. One investigation, conducted by Su Yao, indicated that Fbw7 was down-regulated in non-germinal center B-celllike subtype of DLBCL and low Fbw7 expression was associated with poor prognosis. Overexpression of Fbw7 increased apoptosis rates, meanwhile decreased cell viability [40]. It has been reported that bafilomycin A1 displays anti-cancer effects targeting at apoptosis. For instance, one study states that bafilomycin A1 induces caspase-independent but apoptosis-inducing factor-dependent apoptosis of pediatric B-cell acute lymphoblastic leukemia cells [18]. In the meantime, another study also clarifies that bafilomycin A1 triggers caspase-independent cell death in hepatocellular carcinoma cells, Puma plays a role in bafilomycin A1-mediated cytotoxicity of HCC cells [19]. In agreement with previous studies, we did observe apoptosis in bafilomycin A1 treated DLBCL cells. To our surprise, inconsistent to the above findings, we observed activated caspase- 3 and cleavedParp, which is canonical mechanism of apoptosis. We suggest that bafilomycin A1 may exert different functions at apoptosis under variable cellular contexts.

One of the current strategies in the treatment of cancer is to regulate the levels of autophagy under specific cellular contexts. It's known that autophagy can contribute to therapeutic resistance, while excessive autophagy can cause autophagy-related cell death. For instance, MIR506 induces STAT3-mediated autophagy in pancreatic ductal adenocarcinoma (PDAC) cells, as well as inducing cell death which is dependent on autophagy. MIR506-mediated regulation of the STAT3-BCL2-BECN1 signaling pathway is the major underlying mechanism to induce autophagy-related cell death [41]. Herein, inducing autophagy to cause cell death is one of strategies to regulate autophagy to enhance the treatment of cancer where autophagy plays suppressive roles here. Additionally, another strategy mainly focuses on the relationship between drug resistance with autophagy where autophagy plays cytoprotective roles in cancer [34,42]. One research conducted by $\mathrm{Xi}$ Cheng, revealed that apatinib induces ER stress which is responsible for the induction of autophagy in the treatment of advance gastric carcinoma such as human colorectal cancer (CRC). Furthermore, blocking autophagy enhances the apoptosis in apatinib-treated human CRC cell lines. This provides prospective strategy to relieve apatinib drug resistance by combining apatinib with autophagy inhibitor chloroquine [34].

Thus, we hypothesized that bafilomycin A1 as autophagy inhibitor in high concentration may still inhibit autophagy in low concentration, which is promising strategies if we combine bafilomycin A1 with DLBCL chemotherapy drug rituximab. As anticipated, marked LC3 conversion and accumulation of p62, autolysosomes, increased yellow puncta in ad-mCherry-GFP-LC3 system were all detected in bafilomycin A1-treated DLBCL cells, which revealed that bafilomycin A1 inhibited autophagy flux. Inconsistent to the findings by Yan et al. in bafilomycin A1 treated hepatocellular carcinoma cells in which bafilomycin A1 inhibited the activation of the class I PI3K/Akt/mTOR/p70s6K signaling pathway [19], our data shows that bafilomycin A1 upregulated p-Erk which is the upstream of mTOR, thus p-mTOR also increased. We proposed that bafilomycin A1 may inhibit the early stage of autophagy via inducing the overexpression of p-Erk and p-mTOR 
and inhibit later stage of autophagy by inhibiting the acidification of lysosomes. Herein, we proceeded to combine autophagy inhibitor bafilomycin A1 with DLBCL chemotherapeutic drug rituximab to observe if two drugs can exert synergistic efficacy on DLBCL cells. We found that the concurrent treatment with rituximab synergistically enhanced bafilomycin A1-mediated growth inhibition in DLBCL cell lines. However, the mechanism behind this synergy is unclear. Taken together, our results strongly suggest that bafilomycin A1 can be developed to treat refractory DLBCL. As ongoing efforts on more specific autophagy inhibitors, we anticipate that bafilomycin A1 is a promising candidate drug for the treatment of diffuse large B cell lymphoma.

Author Contributions: Conceptualization, Fang Li and Zhengrong Mao; Data curation, Fang Li; Formal analysis, Fang Li; Funding acquisition, Ren Zhou and Zhengrong Mao; Investigation, Fang Li; Methodology, Fang Li; Project administration, Ren Zhou and Zhengrong Mao; Resources, Fang Li; Software, Fang Li; Validation, Fang Li, Ying Hu and Yizhou Hu; Visualization, Fang Li; Writing - original draft, Fang Li; Writing - review \& editing, Ren Zhou and Zhengrong Mao.

Funding: This research was funded by National Natural Science Foundation of China (No.81071937), the Fundamental Research Funds for the Central Universities (No. 2019QNA7011), Health and Family Planning Commission of Zhejiang Province (No.2018KY362).

\section{Institutional Review Board Statement: Not applicable.}

Informed Consent Statement: Not applicable.

Data Availability Statement: The data presented in this study are available in the article and supplementary material, and are also available on request from the corresponding author.

Conflicts of Interest: The authors declare no conflict of interest.

\section{References}

1. Schmitz, R. et al. Genetics and Pathogenesis of Diffuse Large B-Cell Lymphoma. N Engl J Med 378, 1396-1407, doi:10.1056/NEJMoa1801445 (2018).

2. Liu, Y. \& Barta, S. K. Diffuse large B-cell lymphoma: 2019 update on diagnosis, risk stratification, and treatment. Am J Hematol 94, 604-616, doi:10.1002/ajh.25460 (2019)

3. Gisselbrecht, C. \& Van Den Neste, E. How I manage patients with relapsed/refractory diffuse large B cell lymphoma. Br J Haematol 182, 633-643, doi:10.1111/bjh.15412 (2018).

4. Reddy, A. et al. Genetic and Functional Drivers of Diffuse Large B Cell Lymphoma. Cell 171, 481-494 e415, doi:10.1016/j.cell.2017.09.027 (2017).

5. Kataoka, J. K. K. More accurate prognostic prediction in diffuse large B-cell lymphoma, beyond cell-of-origin. Annals of Oncology, doi:10.1093/annonc/mdy479/5185042 (2018.11).

6. Scott, D. W. et al. Determining cell-of-origin subtypes of diffuse large B-cell lymphoma using gene expression in formalin-fixed paraffin-embedded tissue. Blood 123, 1214-1217, doi:10.1182/blood-2013-11-536433 (2014).

7. Li, S., Young, K. H. \& Medeiros, L. J. Diffuse large B-cell lymphoma. Pathology 50, 74-87, doi:10.1016/j.pathol.2017.09.006 (2018).

8. Kumar, A. et al. Excellent outcomes and lack of prognostic impact of cell of origin for localized diffuse large B-cell lymphoma in the rituximab era. Br J Haematol 171, 776-783, doi:10.1111/bjh.13766 (2015).

9. Tomasetti, C., Li, L. \& Vogelstein, B. Stem cell divisions, somatic mutations, cancer etiology, and cancer prevention. Science 355, 1330-1334, doi:10.1126/science.aaf9011 (2017).

10. Pasqualucci, L. \& Dalla-Favera, R. Genetics of diffuse large B-cell lymphoma. Blood 131, 2307-2319, doi:10.1182/blood-2017-11764332 (2018).

11. Phelan, J. D. et al. A multiprotein supercomplex controlling oncogenic signalling in lymphoma. Nature 560, 387-391, doi:10.1038/s41586-018-0290-0 (2018).

12. Mauvezin, C., Nagy, P., Juhasz, G. \& Neufeld, T. P. Autophagosome-lysosome fusion is independent of V-ATPase-mediated acidification. Nat Commun 6, 7007, doi:10.1038/ncomms8007 (2015).

13. Kozik, P. et al. A human genome-wide screen for regulators of clathrin-coated vesicle formation reveals an unexpected role for the V-ATPase. Nat Cell Biol 15, 50-60, doi:10.1038/ncb2652 (2013).

14. Mauvezin, C. \& Neufeld, T. P. Bafilomycin A1 disrupts autophagic flux by inhibiting both V-ATPase-dependent acidification and Ca-P60A/SERCA-dependent autophagosome-lysosome fusion. Autophagy 11, 1437-1438, doi:10.1080/15548627.2015.1066957 (2015). 
15. Xie, Z. et al. Bafilomycin A1 inhibits autophagy and induces apoptosis in MG63 osteosarcoma cells. Mol Med Rep 10, 1103-1107, doi:10.3892/mmr.2014.2281 (2014).

16. Alexander V. Zhdanov, Ruslan I. Dmitriev, Dmitri B. Papkovsky; Bafilomycin A1 activates HIF-dependent signalling in human colon cancer cells via mitochondrial uncoupling. Biosci Rep 1 December 2012; 32 (6): 587-595. doi: 10.1042/BSR20120085(2012)

17. Lim JH, Park JW, Kim MS, Park SK, Johnson RS, Chun YS. Bafilomycin induces the p21-mediated growth inhibition of cancer cells under hypoxic conditions by expressing hypoxia-inducible factor-1alpha. Mol Pharmacol. 2006;70(6):1856-1865. doi:10.1124/mol.106.028076

18. Yuan, N. et al. Bafilomycin A1 targets both autophagy and apoptosis pathways in pediatric B-cell acute lymphoblastic leukemia. Haematologica 100, 345-356, doi:10.3324/haematol.2014.113324 (2015).

19. Yan $Y$, Jiang K, Liu P, et al. Bafilomycin A1 induces caspase-independent cell death in hepatocellular carcinoma cells via targeting of autophagy and MAPK pathways. Sci Rep. 2016;6:37052. Published 2016 Nov 15. doi:10.1038/srep37052

20. Ohta, T. et al. Bafilomycin A1 induces apoptosis in the human pancreatic cancer cell line Capan-1. J Pathol 185, 324-330, doi:10.1002/(sici)1096-9896(199807)185:3<324::Aid-path72>3.0.Co;2-9 (1998).

21. Oda, K., Nishimura, Y., Ikehara, Y. \& Kato, K. Bafilomycin A1 inhibits the targeting of lysosomal acid hydrolases in cultured hepatocytes. Biochem Biophys Res Commun 178, 369-377, doi:10.1016/0006-291x(91)91823-u (1991).

22. Matsumoto, N. \& Nakanishi-Matsui, M. Proton pumping V-ATPase inhibitor bafilomycin A1 affects Rab7 lysosomal localization and abolishes anterograde trafficking of osteoclast secretory lysosomes. Biochem Biophys Res Commun 510, 421-426, doi:10.1016/j.bbrc.2019.01.118 (2019).

23. Levine, B. \& Kroemer, G. Biological Functions of Autophagy Genes: A Disease Perspective. Cell 176, 11-42, doi:10.1016/j.cell.2018.09.048 (2019).

24. Mizushima, N., Levine, B., Cuervo, A. M. \& Klionsky, D. J. Autophagy fights disease through cellular self-digestion. Nature 451, 1069-1075, doi:10.1038/nature06639 (2008).

25. Ktistakis, N. T. Who plays the ferryman: ATG2 channels lipids into the forming autophagosome. J Cell Biol 218, 1767-1768, doi:10.1083/jcb.201904159 (2019).

26. Singh, S. S. et al. Dual role of autophagy in hallmarks of cancer. Oncogene 37, 1142-1158, doi:10.1038/s41388-017-0046-6 (2018).

27. Klionsky, D. J. et al. Guidelines for the use and interpretation of assays for monitoring autophagy (3rd edition). Autophagy 12, 1-222, doi:10.1080/15548627.2015.1100356 (2016).

28. Joshi, S. \& Ryan, K. M. Autophagy chews Fap to promote apoptosis. Nat Cell Biol 16, 23-25, doi:10.1038/ncb2899 (2014).

29. Ding, X. et al. RAB2 regulates the formation of autophagosome and autolysosome in mammalian cells. Autophagy 15, 17741786, doi:10.1080/15548627.2019.1596478 (2019).

30. Levine, G. K. a. B. Autophagic cell death, the story of a misnomer. Nat Rev Mol Cell Biol., doi:10.1038/nrm2527 (2008 December).

31. Dower, C. M., Wills, C. A., Frisch, S. M. \& Wang, H. G. Mechanisms and context underlying the role of autophagy in cancer metastasis. Autophagy 14, 1110-1128, doi:10.1080/15548627.2018.1450020 (2018).

32. Yang, Z. \& Klionsky, D. J. Eaten alive: a history of macroautophagy. Nat Cell Biol 12, 814-822, doi:10.1038/ncb0910-814 (2010).

33. Shen, H. M. \& Codogno, P. Autophagic cell death: Loch Ness monster or endangered species? Autophagy 7, 457-465, doi:10.4161/auto.7.5.14226 (2011).

34. Cheng, X. et al. Targeting autophagy enhances apatinib-induced apoptosis via endoplasmic reticulum stress for human colorectal cancer. Cancer Lett 431, 105-114, doi:10.1016/j.canlet.2018.05.046 (2018).

35. Bryant, K. L. et al. Combination of ERK and autophagy inhibition as a treatment approach for pancreatic cancer. Nat Med 25, 628-640, doi:10.1038/s41591-019-0368-8 (2019).

36. Sbrissa, D., Naisan, G., Ikonomov, O. C. \& Shisheva, A. Apilimod, a candidate anticancer therapeutic, arrests not only PtdIns(3,5)P2 but also PtdIns5P synthesis by PIKfyve and induces bafilomycin A1-reversible aberrant endomembrane dilation. PLoS One 13, e0204532, doi:10.1371/journal.pone.0204532 (2018).

37. Inoue, S., Setoyama, Y. \& Odaka, A. Phagocytosis of bafilomycin A1-treated apoptotic neuroblastoma cells by bone marrowderived dendritic cells initiates a CD8alpha+ lymphocyte response to neuroblastoma. J Pediatr Hematol Oncol 36, e290-295, doi:10.1097/mph.0000000000000060 (2014).

38. Teplova, V. V., Tonshin, A. A., Grigoriev, P. A., Saris, N. E. \& Salkinoja-Salonen, M. S. Bafilomycin A1 is a potassium ionophore that impairs mitochondrial functions. J Bioenerg Biomembr 39, 321-329, doi:10.1007/s10863-007-9095-9 (2007).

39. Li, L. Q., Xie, W. J., Pan, D., Chen, H. \& Zhang, L. Inhibition of autophagy by bafilomycin A1 promotes chemosensitivity of gastric cancer cells. Tumour Biol 37, 653-659, doi:10.1007/s13277-015-3842-z (2016).

40. Yao S, Xu F, Chen Y, et al. Fbw7 regulates apoptosis in activated B-cell like diffuse large B-cell lymphoma by targeting Stat3 for ubiquitylation and degradation. J Exp Clin Cancer Res. 2017;36(1):10. Published 2017 Jan 10. doi:10.1186/s13046-016-0476-y

41. Sun L, Hu L, Cogdell D, et al. MIR506 induces autophagy-related cell death in pancreatic cancer cells by targeting the STAT3 pathway. Autophagy. 2017;13(4):703-714. doi:10.1080/15548627.2017.1280217

42 Kinsey, C. G. et al. Protective autophagy elicited by RAF-->MEK-->ERK inhibition suggests a treatment strategy for RASdriven cancers. Nat Med 25, 620-627, doi:10.1038/s41591-019-0367-9 (2019).

42. Kinsey, C. G. et al. Protective autophagy elicited by RAF-->MEK-->ERK inhibition suggests a treatment strategy for RAS-driven cancers. Nat Med 25, 620-627, doi:10.1038/s41591-019-0367-9

(2019). 